Article

\title{
Does the Number of Fingers on the Bag Influence Volume Delivery? A Randomized Model Study of Bag-Valve-Mask Ventilation in Infants
}

\author{
David Zweiker ${ }^{1}{ }^{(0)}$, Hanna Schwaberger ${ }^{1,2}$, Berndt Urlesberger ${ }^{1}$, Lukas P Mileder ${ }^{1}(\mathbb{D}$, \\ Nariae Baik-Schneditz ${ }^{1}$, Gerhard Pichler ${ }^{1}$, Georg M Schmölzer ${ }^{1,2,3,4} \oplus$ and \\ Bernhard Schwaberger 1,2,*(D) \\ 1 Division of Neonatology, Department of Pediatrics and Adolescent Medicine, Medical University of Graz, \\ Graz 8010, Austria; david.zweiker@medunigraz.at (D.Z.); hanna.kilbertus@gmail.com (H.S.); \\ berndt.urlesberger@medunigraz.at (B.U.); lukas.mileder@medunigraz.at (L.P.M.); \\ nariae.baik@medunigraz.at (N.B.-S.); gerhard.pichler@medunigraz.at (G.P.); \\ georg.schmoelzer@me.com (G.M.S.) \\ 2 Medizinercorps Graz, Austrian Red Cross Federal Association Styria, Graz 8010, Austria \\ 3 Centre for the Studies of Asphyxia and Resuscitation, Neonatal Research Unit, Royal Alexandra Hospital, \\ Edmonton, AB T5H 3V9, Canada \\ 4 Department of Pediatrics, University of Alberta, Edmonton, AB T6G 1C9, Canada \\ * Correspondence: bernhard.schwaberger@medunigraz.at; Tel.: +43-316-385-30018
}

Received: 13 August 2018; Accepted: 17 September 2018; Published: 21 September 2018

\begin{abstract}
We sought to compare the effectiveness of two versus five fingers used for bag-valve-mask $(B V M)$ ventilation on effective tidal volume $\left(V_{\text {Teff }}\right)$ delivery in an infant resuscitation model. In a randomised cross-over study, 40 healthcare professionals ventilated a modified leak-free infant resuscitation manikin with both two and five fingers, using a self-inflating bag. The delivered and effective tidal volumes, ventilation rate, and mask leak were measured and recorded using a respiratory function monitor. We found no significant differences in the $\mathrm{V}_{\text {Teff }}$ (five-finger $61.7 \pm 23.9$ vs. two-finger $58.8 \pm 16.6 \mathrm{~mL} ; p=0.35)$ or ventilatory minute volume $(2.71 \pm 1.59$ vs. $2.76 \pm 1.24 \mathrm{~L} / \mathrm{min} ; p=0.40$ ) of both BVM ventilation techniques. However, there was an increase in the delivered tidal volume $\left(\mathrm{V}_{\mathrm{Tdel}}\right)$ and mask leak when using the five-finger technique compared with the two-finger technique $\left(\mathrm{V}_{\text {Tdel }} 96.1 \pm 19.4\right.$ vs. $87.7 \pm 15.5 \mathrm{~mL} ; p<0.01$; and mask leak $34.6 \pm 23.0$ vs. $30.0 \pm 21.0 \% ; p=0.02)$. Although the five-finger technique was associated with an increased mask leak, the number of fingers used during the BVM ventilation had no effect on $\mathrm{V}_{\text {Teff }}$ in an infant resuscitation model.
\end{abstract}

Keywords: paediatric resuscitation; ventilation; non-invasive; mask ventilation; bag-valve-mask ventilation; ventilation technique

\section{Introduction}

Respiratory emergencies leading to hypoxaemia are the most frequent cause of pediatric cardiac arrest [1,2]. Furthermore, approximately $10 \%$ of newborn infants require respiratory support at birth [3,4]. Thus, the cornerstone of successful resuscitation in the pediatric population is adequate ventilation [5,6]. Bag-valve-mask ventilation (BVM) with self-inflating bags (SIBs) is the recommended first line of therapy for airway control and ventilation in children [5]. SIBs are widely used for pediatric and neonatal resuscitation, especially outside of the hospital or in resource-limited settings [7,8].

Healthcare personnel commonly provide excessive ventilation during cardio-pulmonary resuscitation $[9,10]$, and this may be harmful [5]. Therefore, the correct BVM ventilation technique 
is essential in order to avoid hypo- and hyper-ventilation. For guidance, the current resuscitation guidelines mention adequate chest rise, mask holding techniques, and a correct head position during mask ventilation for newborn infants [5,6]. However, an optimal technique for holding the SIB is not provided. In pediatric emergency care courses, questions regarding the number of fingers that are to be used on the SIB for the delivery of an adequate tidal volume $\left(\mathrm{V}_{\mathrm{T}}\right)$ are often raised. Currently, there is only anecdotal evidence about the most effective holding technique when using SIBs. Current neonatal and pediatric emergency literature describes a "rule of thumb", recommending the use of the thumb and one additional finger per kilogram body weight when using a $250 \mathrm{~mL}$ SIB [11-13]. Furthermore, a popular German midwifery book recommends the use of the thumb, index finger, and middle finger during BVM ventilation [14]. This diversity of recommendations leads to the uncertainty of healthcare personnel in training about the optimal number of fingers needed to hold a SIB. We aimed to examine and compare two different SIB holding techniques during a simulated resuscitation on an infant model. We hypothesized that the number of fingers used during BVM ventilation had an effect on the $\mathrm{V}_{\mathrm{T}}$ delivery. Additionally, we were interested in the influence of the participants' anthropometric parameters on the $\mathrm{V}_{\mathrm{T}}$ delivery.

\section{Materials and Methods}

\subsection{Participants}

A randomized controlled pilot cross-over study was performed using 40 healthcare professionals. The Regional Committee on Biomedical Research Ethics of the Medical University of Graz (26-582 ex 13/14) approved the study. Pediatric intensive care nurses from the University Hospital at the Medical University of Graz, Austria, and medical students volunteering as paramedics were invited to participate in the study. The registered nurses were all trained in neonatal and pediatric resuscitation according to European Resuscitation Council (ERC) guidelines [5,6]. The participating paramedics had all completed at least three years of medical school education at the time of participation, and had received advanced emergency medical technician training [15], as well as at least three weeks of pediatric airway management training during a clinical elective at the Department of Pediatric Anesthesia [16]. We included all participants with informed consent into the study, and an absence thereof was the theoretical exclusion criterion.

The present study was designed as a pilot study, because no data on sensitivity and specificity were available. Therefore, no sample size calculation was performed. We hypothesized that a sample size of 40 would be representative for the present study [17].

\subsection{Study Protocol}

The participants acted as their own control and were asked to perform adequate ventilation for $90 \mathrm{~s}$ each. By throwing dice, the participants were randomized to either use two or five fingers first. For the two-finger technique, participants were instructed to use their thumb and index finger. For the five-finger technique, the SIB had to be compressed with the whole hand. Between both of the evaluation sessions, the participants were allowed to rest for five minutes so as to reduce the potential bias due to fatigue. No verbal feedback was given to the participants.

\subsection{Manikin}

A Laerdal Resusci Baby manikin (Laerdal Medical, Stavanger, Norway) was modified by removing the lung and stomach bags, and positioning a $0.5 \mathrm{~L}$ test lung (Dräger, Lubeck, Germany) into the chest, so that the chest excursions were similar to a standard training manikin. The test lung was connected to the mouth with non-distensible tubing and an airtight seal. When pressurized to $30 \mathrm{~cm}$ $\mathrm{H}_{2} \mathrm{O}$, the maximal lung volume in our infant model was approximately $90 \mathrm{~mL}$. 


\subsection{Ventilation Device}

The Ambu Baby R Resuscitator (Ambu, Ballerup, Denmark), a SIB with a maximal tidal volume of approximately $300 \mathrm{~mL}$, was used. A silicon mask size 0/1 (Laerdal Medical, Stavanger, Norway) was connected to the SIB. The complete set-up is presented in Figure 1.

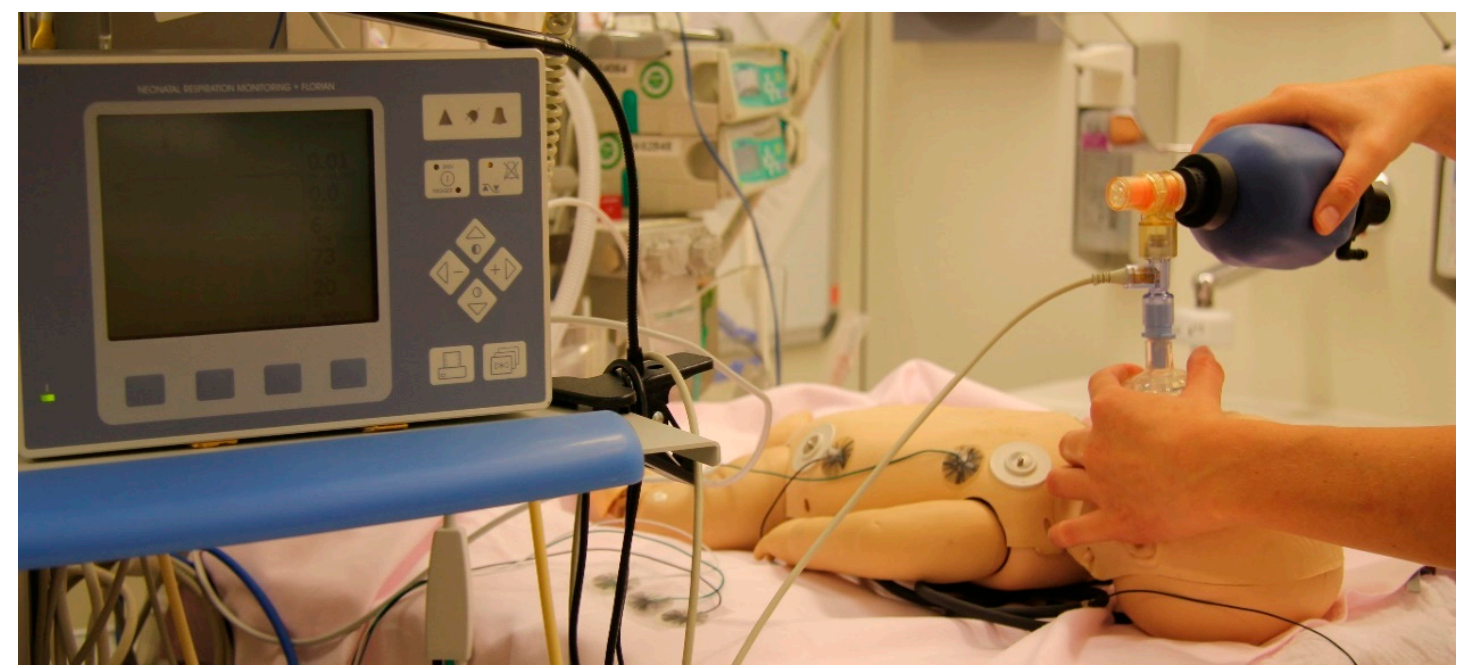

Figure 1. The experimental set-up consisted of a modified leak-free baby manikin, a self-inflating bag, and a respiratory function monitor.

\subsection{Respiratory Function Monitor (RFM)}

A Florian Neonatal RFM (Acutronic Medical Systems, Zug, Switzerland) was used to measure the ventilation parameters. A hot-wire anemometer flow sensor was placed between the mask and SIB. The $\mathrm{V}_{\mathrm{T}}$ was calculated by integrating the flow signals. The mask leak was expressed as a percentage of the inspired $\mathrm{V}_{\mathrm{T}}$. During the recordings, participants were blinded to the screen of the RFM.

\subsection{Data Acquisition and Analysis}

The gas flow, $\mathrm{V}_{\mathrm{T}}$, and airway pressure data were recorded at $200 \mathrm{~Hz}$ using a computer with $\alpha$-Trace Digital MM (Best Medical Systems, Vienna, Austria) multi-channel system. We collected the gender, hand size (palmar distance from os pisiforme to distal end of second index finger), and shoe size (Continental European system) of the participants anonymously. Shoe size was evaluated in order to prove its potential use as a surrogate parameter for hand size.

\subsection{Statistical Analysis}

We primarily aimed to investigate the effect of a two- vs. five-finger BVM ventilation techniques on effective tidal volume $\left(\mathrm{V}_{\text {Teff }}\right)$. To account for any possible problems at the beginning of each ventilation session, only the last $60 \mathrm{~s}$ of the recorded $90 \mathrm{~s}$ were analysed. We first calculated each respiratory cycle and then the mean for each participant for the following parameters: the delivered tidal volume $\left(\mathrm{V}_{\text {Tdel }}\right)(\mathrm{mL})$, total inspiratory volume at the $B V M, \mathrm{~V}_{\text {Teff }}(\mathrm{mL})$, total expiratory volume at the BVM, and mask leak $(\%)$, as $\frac{V_{\text {Tdel }}-T_{\text {Teff }}}{V_{\text {Tdel }}}$. Furthermore, we calculated the ventilation rate $\left(\mathrm{min}^{-1}\right)$ and ventilatory minute volume $(\mathrm{L} / \mathrm{min})$ as follows: $\mathrm{V}_{\text {Teff }} *$ ventilation rate.

We expressed values as the mean \pm standard deviation, median (interquartile range), or count (proportion), wherever appropriate. Firstly, we performed the Spearman correlation and non-parametric testing for the possible bivariate association between the assessed parameters. Then, we analysed the influence of the baseline parameters (gender, hand size, shoe size, and pediatric intensive care nurses vs. paramedics) on $\mathrm{V}_{\text {Teff }}$ using the two-finger technique vs. the five-finger technique by general linear modelling. 
To guide future trials, we performed a post hoc sample size calculation using $G^{*}$ Power (Heinrich Heine University, Düsseldorf, Germany) to calculate the number of individuals needed for the measurement of a relevant difference in $\mathrm{V}_{\text {Tdel }}$, which was considered as a difference of at least $15 \%$ between both methods. A two-sided $p$-value of $<0.05$ was considered significant. We used IBM ${ }^{\circledR}$ SPSS 20 (Armonk, NY, USA) for the data analysis.

The whole study was conducted from September to October 2014 at the Medical University of Graz, Graz, Austria. We did not change the protocol or the definition of the outcome measures during or after the conduction of the study.

\section{Results}

A flow diagram can be found in Figure 2. There were 20 pediatric intensive care nurses and 20 paramedics that participated in the study. Of the participants, $25(62.5 \%)$ were female, with an overall mean shoe size of $40.0 \pm 2.7$ Paris points and a median hand size of $17.5(17-18.5) \mathrm{cm}$. A total of 5401 inflations were analysed, including 2796 inflations with two fingers and 2605 inflations with five fingers. All of the participants completed the entire protocol.

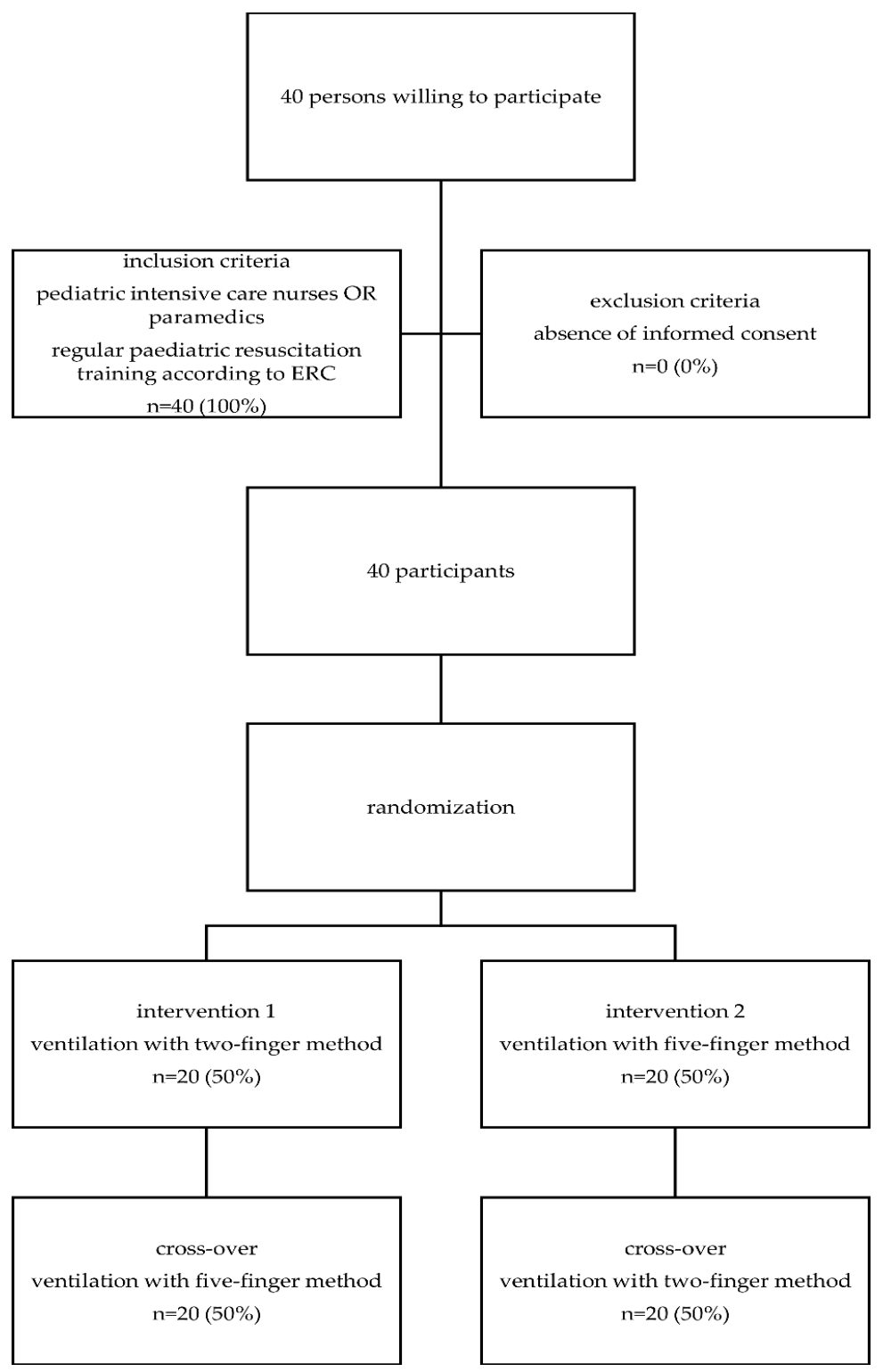

Figure 2. Study flow diagram. ERC-European Resuscitation Council. 


\subsection{Effective and Delivered Tidal Volume $\left(V_{\text {Teff }}\right.$ and $\left.V_{\text {Tdel }}\right)$}

The $\mathrm{V}_{\text {Tdel }}$ was significantly lower during the two-finger technique compared with the five-finger technique ( $87.7 \pm 15.5$ vs. $96.1 \pm 19.4 \mathrm{~mL}$, respectively; $p<0.01$; as seen in Table 1$)$. The mask leak was also significantly reduced for the two-finger technique ( $27.9 \pm 22.4$ vs. $34.4 \pm 24.5 \mathrm{~mL} ; p=0.02)$. As a result, the $\mathrm{V}_{\text {Teff }}$ did not differ significantly between the two-finger and five-finger techniques (58.8 \pm 16.6 vs. $61.7 \pm 23.9 \mathrm{~mL}$, respectively; $p=0.35$; Table 1 ). $\mathrm{V}_{\text {Teff }}$ was similar in the pediatric intensive care nurses and paramedics. The difference between two- and five-finger techniques tended to be more pronounced at higher tidal volumes, but this correlation was not significant (Spearman's $\rho$ 0.24; $p=0.14 ;$ Figure 3).

Table 1. Comparison of tidal volumes, leak, and ventilation rate between two-finger vs. five-finger techniques.

\begin{tabular}{ccccc}
\hline & $\begin{array}{c}\text { Two-Finger } \\
\text { Technique }\end{array}$ & $\begin{array}{c}\text { Five-Finger } \\
\text { Technique }\end{array}$ & $\begin{array}{c}\text { Absolute } \\
\text { Difference }\end{array}$ & $p$-Value \\
\hline $\mathrm{V}_{\text {Teff }}(\mathrm{mL})$ & $58.8 \pm 16.6$ & $61.7 \pm 23.9$ & $+2.9 \pm 15.9$ & 0.35 \\
$\mathrm{~V}_{\text {Tdel }}(\mathrm{mL})$ & $87.7 \pm 15.5$ & $96.1 \pm 19.4$ & $+9.4 \pm 12.7$ & $<0.01^{*}$ \\
Mask leak $(\mathrm{mL})$ & $27.9 \pm 22.4$ & $34.4 \pm 24.5$ & $+6.5 \pm 16.7$ & $0.022^{*}$ \\
Mask leak $(\%)$ & $30.0 \pm 21.0$ & $34.6 \pm 23.0$ & $+4.5 \pm 16.0$ & $0.02^{*}$ \\
Ventilation rate (min \\
Ventilatory minute & $46.6 \pm 16.9$ & $43.4 \pm 16.4$ & $-3.2 \pm 6.1$ & $<0.01^{*}$ \\
volume $(\mathrm{L} / \mathrm{min})$ & $2.76 \pm 1.24$ & $2.71 \pm 1.59$ & $-0.05 \pm 0.71$ & 0.40 \\
\hline
\end{tabular}

$\mathrm{V}_{\text {Teff }}$-effective tidal volume; $\mathrm{V}_{\text {Tdel }}$ - delivered tidal volume; ${ }^{*} p<0.05$.

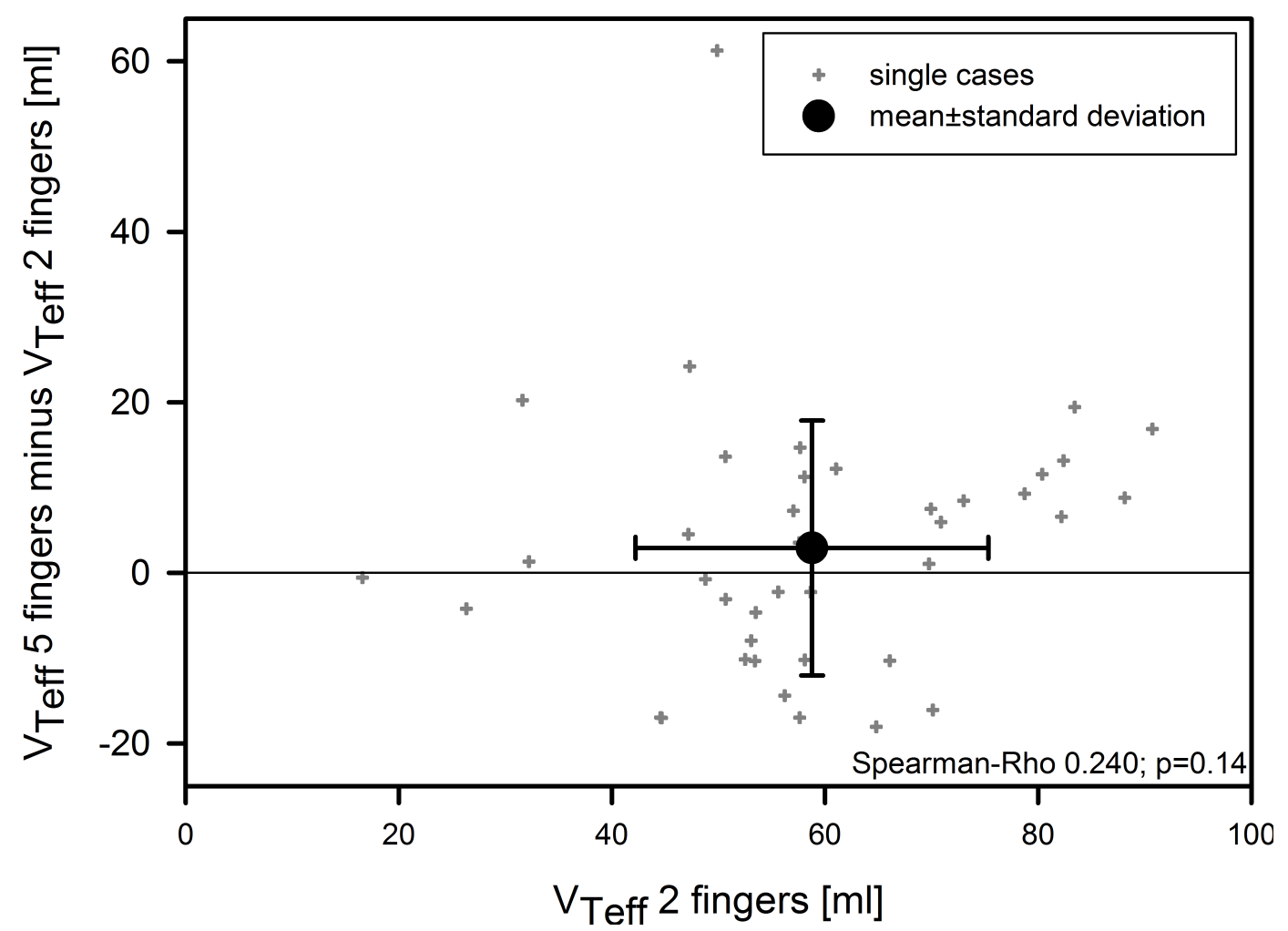

Figure 3. Comparison of effective tidal volume $\left(\mathrm{V}_{\text {Teff }}\right)$ between two-finger ventilation and five-finger ventilation.

\subsection{Ventilation Rate and Ventilatory Minute Volume}

We observed a statistically significant difference with higher ventilation rates for the two-finger technique compared with the five-finger technique ( $46.6 \pm 16.9$ vs. $43.4 \pm 16.4 \mathrm{~min}^{-1}$, respectively; 
$p<0.01)$. The minute ventilation was similar in both groups $(2.76 \pm 1.24 \mathrm{vs.} 2.71 \pm 1.59 \mathrm{~L} / \mathrm{min}$, respectively; absolute difference $0.05 \pm 0.71 \mathrm{~L} / \mathrm{min}$, respectively; $p=0.40$ ).

\subsection{Influence of Anthropometric Data on $V_{\text {Teff }}$}

The correlations of the hand and shoe sizes with $\mathrm{V}_{\text {Teff }}$ were low and non-significant $(\rho<0.1$ and $p>0.1$ for both).

\subsection{Multivariate Analysis for $V_{\text {Teff }}$}

We did not find any significant interactions between the baseline parameters (gender, hand size, shoe size, and pediatric intensive care nurses vs. paramedics) and our primary endpoint $\left(\mathrm{V}_{\text {Teff }}\right)$.

\subsection{Post-Hoc Sample Size Calculation}

As the mean $\mathrm{V}_{\text {Teff }}$ in the two-finger technique was $58.8 \mathrm{~mL}$, the mean $\mathrm{V}_{\text {Teff }}$ would have to be at least $67.6 \mathrm{~mL}$ in the five-finger technique in order to be clinically relevant. We performed a post hoc sample-size calculation with a normalized effect size of 0.481 (considering a standard deviation of $20 \mathrm{~mL}$ ). To achieve a power of $80 \%$, a sample size of at least 36 participants would be necessary to detect a relevant difference between both methods.

\section{Discussion}

To the best of our knowledge, this is the first study comparing the number of fingers used during mask ventilation using a SIB, and their effect on $\mathrm{V}_{\mathrm{T}}$ delivery. We did not observe any differences in $\mathrm{V}_{\text {Teff }}$ between the two-finger and five-finger technique during mask ventilation. Our results support the current ERC guidelines, which do not offer any specific recommendations regarding the number of fingers used for mask ventilation $[5,6,18]$. The ERC guidelines only state that insufflations should result in a chest rise so as to ensure adequate $V_{T}$ delivery. Other recommendations, such as the aforementioned "rule of thumb", lack any scientific evidence and should therefore not be used. Healthcare personnel in training may therefore be taught to use as many fingers as they want to on their SIB, as long as the clinical features signalize adequate ventilation.

Although the five-finger technique may not necessarily lead to a higher $\mathrm{V}_{\text {Teff }}$, the mask leak and $\mathrm{V}_{\text {Tdel }}$ were shown to be higher compared with the two-finger technique. We assume that the ventilation pressures during the BVM ventilation are higher using the five-finger technique, and therefore result in a higher mask leak. By recognizing an adequate chest rise early on, healthcare professionals may interrupt the inflation immediately, and thus avoid an overinflation of the lungs, even when delivering higher inspiration pressures.

We found statistically significant differences in the ventilation rates between the two-finger and five-finger techniques. This finding may be of questionable clinical relevance because of the small absolute difference, especially when considering that the values were within the normal range of the current guideline recommendations in both groups.

Besides analysing the effect of the number of fingers used, we studied the effect of anthropometric measures and gender on tidal volumes. In certain resuscitation situations during our clinical careers, we had the impression that men with large hands (and feet) would deliver higher tidal volumes during neonatal or infant resuscitation. However, this hypothesis needs to be rejected because of a negligible correlation between hand/shoe size and $\mathrm{V}_{\text {Teff }}$ in our study.

\section{Limitations}

Although we tried to design our simulation model to be as realistic as possible, and the study was large enough to detect clinically relevant differences, the results may not fully reflect a real clinical setting. As a result of the modifications to our resuscitation manikin in order to ensure air tightness, the absolute $\mathrm{V}_{\text {Teff }}$ in our model was significantly higher and cannot be compared to the tidal volumes 
in actual neonates or infants. However, we could determine changes in the tidal volumes between both of the ventilation techniques easily, because each volunteer served as his/her own control.

Furthermore, in this study, we only assessed BVM ventilation. Therefore, our results may not be transferable to invasive ventilation or non-invasive ventilation using a T-piece or another ventilatory device.

Thirdly, we analyzed BVM ventilation in the participants that received regular training in neonatal and/or pediatric resuscitation. Therefore, this study is not generalizable to other personnel who are trained less often.

Lastly, we aimed to minimize dead space, and therefore did not assess the differences in pressure between both techniques, which may also have had major implications on the clinical outcome.

\section{Conclusions}

This study shows that, in an infant model, the use of two or five fingers for BVM ventilation leads to a similar $\mathrm{V}_{\mathrm{T}}$ delivery, regardless of the hand or shoe size of the performing personnel.

Author Contributions: Conceptualization, H.S., B.U., G.P., G.M.S. and B.S.; data curation, H.S. and B.S.; formal analysis, H.S. and B.S.; funding acquisition, G.M.S. and B.S.; investigation, H.S. and B.S.; methodology, H.S., B.U., L.P.M., N.B.-S. and B.S.; project administration, B.S.; resources, B.S.; software, D.Z., H.S. and B.S.; supervision, B.S.; validation, D.Z., H.S. and B.S.; visualization, D.Z. and B.S.; writing-original draft, D.Z.; writing-review and editing, H.S., B.U., L.P.M., N.B.-S., G.P., G.M.S. and B.S.

Funding: We would like to thank the public for the donation of money to our funding agencies. G.M.S. is a recipient of the Heart and Stroke Foundation/University of Alberta Professorship of Neonatal Resuscitation; a National New Investigator of the Heart and Stroke Foundation, Canada; and an Alberta New Investigator of the Heart and Stroke Foundation Alberta. The sponsors of the study had no role in the study design, data collection, data analysis, data interpretation, or writing of the report.

Acknowledgments: We want to thank all volunteers who participated in this study.

Conflicts of Interest: The authors declare no conflict of interest.

\section{References}

1. Reis, A.G.; Nadkarni, V.; Perondi, M.B.; Grisi, S.; Berg, R.A. A prospective investigation into the epidemiology of in-hospital pediatric cardiopulmonary resuscitation using the international utstein reporting style. Pediatrics 2002, 109, 200-209. [CrossRef] [PubMed]

2. Tham, L.P.; Chan, I. Paediatric out-of-hospital cardiac arrests: Epidemiology and outcome. Singap. Med. J. 2005, 46, 289-296.

3. Kattwinkel, J.; Niermeyer, S.; Nadkarni, V.; Tibballs, J.; Phillips, B.; Zideman, D.; Van Reempts, P.; Osmond, M. Resuscitation of the newly born infant: An advisory statement from the pediatric working group of the international liaison committee on resuscitation. Resuscitation 1999, 40, 71-88. [CrossRef]

4. Singhal, N.; McMillan, D.D.; Yee, W.H.; Akierman, A.R.; Yee, Y.J. Evaluation of the effectiveness of the standardized neonatal resuscitation program. J. Perinatol. 2001, 21, 388-392. [CrossRef] [PubMed]

5. Maconochie, I.K.; Bingham, R.; Eich, C.; Lopez-Herce, J.; Rodriguez-Nunez, A.; Rajka, T.; Van de Voorde, P.; Zideman, D.A.; Biarent, D.; Paediatric Life Support Section Collaborators. European resuscitation council guidelines for resuscitation 2015: Section 6. Paediatric life support. Resuscitation 2015, 95, 223-248. [CrossRef] [PubMed]

6. Wyllie, J.; Bruinenberg, J.; Roehr, C.C.; Rudiger, M.; Trevisanuto, D.; Urlesberger, B. European resuscitation council guidelines for resuscitation 2015: Section 7. Resuscitation and support of transition of babies at birth. Resuscitation 2015, 95, 249-263. [CrossRef] [PubMed]

7. O'Donnell, C.P.; Davis, P.G.; Morley, C.J. Positive pressure ventilation at neonatal resuscitation: Review of equipment and international survey of practice. Acta Paediatr. 2004, 93, 583-588. [CrossRef] [PubMed]

8. Thallinger, M.; Ersdal, H.L.; Ombay, C.; Eilevstjonn, J.; Stordal, K. Randomised comparison of two neonatal resuscitation bags in manikin ventilation. Arch. Dis. Child. Fetal Neonatal Ed. 2016, 101, F299-F303. [CrossRef] [PubMed] 
9. Donoghue, A.; Hsieh, T.C.; Myers, S.; Mak, A.; Sutton, R.; Nadkarni, V. Videographic assessment of cardiopulmonary resuscitation quality in the pediatric emergency department. Resuscitation 2015, 91, 19-25. [CrossRef] [PubMed]

10. Niebauer, J.M.; White, M.L.; Zinkan, J.L.; Youngblood, A.Q.; Tofil, N.M. Hyperventilation in pediatric resuscitation: Performance in simulated pediatric medical emergencies. Pediatrics 2011, 128, e1195-e1200. [CrossRef] [PubMed]

11. Hannsmann, G. Basics in cardiopulmonary resuscitation of newborn infants. In Neonatal Emergencies; Hannsmann, G., Ed.; University Press: Cambridge, UK, 2009; p. 74.

12. Hansmann, G.; Humpl, T.; Zimmermann, A. Neonatal-emergencies: Basics in cardiopulmonary resuscitation. Zeitschrift fur Geburtshilfe und Neonatologie 2004, 208, 43-56. [CrossRef] [PubMed]

13. Strauss, A. Erstversorgung nach der geburt. In Geburtshilfe Basics; Springer: Heidelberg, Germany, 2006; p. 427.

14. Hebammen, B.D. Das Neugeborene in der Hebammenpraxis; Thieme: Stuttgart, Germany, 2004.

15. Weninger, P.; Hertz, H.; Mauritz, W. International ems: Austria. Resuscitation 2005, 65, 249-254. [CrossRef] [PubMed]

16. Prause, G.; Oswald, S.; Himler, D.; Wildner, G.; Gemes, G. The medizinercorps graz: A 120-year-old institution of emergency medicine. Prehosp. Emerg. Care 2013, 17, 416-420. [CrossRef] [PubMed]

17. Thabane, L.; Ma, J.; Chu, R.; Cheng, J.; Ismaila, A.; Rios, L.P.; Robson, R.; Thabane, M.; Giangregorio, L.; Goldsmith, C.H. A tutorial on pilot studies: The what, why and how. BMC Med. Res. Methodol. 2010, 10, 1. [CrossRef] [PubMed]

18. Ambu. Mark IV Baby Manual; Ambu: Ballerup, Denmark, 2005; p. 5.

(C) 2018 by the authors. Licensee MDPI, Basel, Switzerland. This article is an open access article distributed under the terms and conditions of the Creative Commons Attribution (CC BY) license (http:/ / creativecommons.org/licenses/by/4.0/). 